\title{
Multiagent Coordination Enabling Autonomous Logistics
}

Dr.-Ing. Arne Schuldt, wissenschaftlicher Mitarbeiter, Technologie-Zentrum Informatik und Informationstechnik (TZI), Universität Bremen

\begin{abstract}
Supply network management is a challenging task due to the complexity, the dynamics, and the distribution of logistics processes. Automated process control thus requires to reduce the computational complexity and to cope with the dynamics locally. The paradigm of autonomous control in logistics means that control of logistics processes is delegated to the participating objects. As an example, shipping containers may themselves plan and schedule their way through logistics networks in accordance with objectives imposed by their owners. This work (Schuldt, 2010) solves the implementation of autonomous control with multiagent technology. This multiagent-based solution has been used in a realistic simulation of the container logistics processes of Tchibo. The validation shows that autonomous control is actually possible and that it outperforms the previous centralised dispatching approach by significantly increasing the resource utilisation efficiency. Moreover, the multiagent system relieves human dispatchers from dealing with standard cases, giving them more time to solve exceptional cases appropriately.
\end{abstract}

\section{Logistics Requirements}

Supply network management becomes increasingly complex, dynamic, and distributed, e. g., due to the globalisation and the goods structure effect. Due to these challenges, conventional centralised process control turns out to be feasible only for particular applications (Bretzke, 2008). The high number of logistics objects as well as their manifold parameters induces a high computational effort of process control. In addition, the dynamics of logistics processes can render optimal plans outdated already in the moment their generation is finished. Furthermore, the spatial distribution of logistics processes often prevents information from being available centrally on time. Central control that tries to generate optimal plans for a complete logistics system is thus frequently not feasible. 
The paradigm of autonomous logistics (Hülsmann \& Windt, 2007) aims at automating process control by delegating decision-making to the participating logistics objects. Based on objectives imposed by their owners, these autonomous logistics entities can themselves plan and schedule their way through logistics networks. Each entity incorporates only its own parameters as well as those of cooperating entities. Therefore, the computational complexity can be reduced significantly and dynamics can be dealt with locally. This vision requires not only granting the autonomy, but also delegating the ability to make decisions to the logistics objects. New identification, localisation, sensor, communication, and data processing technologies enable logistics objects, such as shipping containers, to make decisions on their own. This work solves the implementation of decision-making by local data processing as well as the coordination of the individual autonomous logistics entities, therewith enabling the application to real-world process control.

\section{Multiagent-Based Approach}

Multiagent systems derived from Distributed Artificial Intelligence have been identified as an appropriate method to implement autonomous control in logistics. Characteristics of intelligent agents are that they are autonomous, that they act both reactively and pro-actively, and that they are able to communicate and to cooperate with each other (Wooldridge, 1999). These properties directly reflect the requirements for autonomous logistics entities. Both logistics service providers and consumers can be modelled with intelligent agents.

Despite their autonomy, however, single logistics objects can hardly satisfy their objectives in isolation. The identified reasons that advocate cooperation among them are twofold. On the one hand, joint actions of objects with similar goals significantly reduce the interaction effort, thereby preventing that the reduction in computational complexity is outweighed. On the other hand, autonomous logistics entities can only jointly meet minimum utilisation requirements of logistics service providers. Hence, cooperation among logistics objects is an important prerequisite for autonomous control in logistics.

A well-known concept in Distributed Artificial Intelligence is the model for cooperation by Wooldridge and Jennings (1999). It formally specifies the steps required for agent cooperation, starting from the identification of a potential for cooperation, over team and plan formation, to joint team action. This concept, however, is rather general and thus abstract which means that particular interaction schemes 
are underspecified. Based on these general steps, this thesis develops specific interaction schemes that are required for autonomous logistics.

Three different interaction protocols for team formation of autonomous logistics entities are proposed. The protocols are based on a directory, a broker, and multicast messaging, respectively. Their most distinguishing properties are the degree of decentralisation as well as the interaction effort to be spent for team formation. The interaction effort increases with the degree of decentralisation. Based on this finding, the potential as well as limitations for autonomous logistics are derived analytically. Regarding the team formation mechanisms, this analysis allows for choosing the least complex interaction schemes for a specific application in logistics.

Team formation is the foundation for jointly coordinating the primary logistics functions transport, handling, storage, and picking. Furthermore, interaction schemes for plan formation and team action are developed. This is accomplished in three steps. Firstly, the allocation of logistics services by individual autonomous logistics entities is operationalised. Secondly, this approach is extended to the joint allocation of logistics resources by teams of autonomous logistics entities. Finally, also the intra-agent coordination of multiple logistics functions is solved. This allows for coordinating multiple primary logistics functions to satisfy complex logistics objectives. Therewith, this thesis provides a complete implementation of the general model for cooperation in real-world applications of autonomous control in logistics.

\section{Application and Evaluation}

The approach is validated in the context of real-world scheduling processes. As a basis, a comprehensive case study of the procurement logistics processes of Tchibo has been conducted. Tchibo is a major European retailer of consumer products with more than 1,200 own shops and over 56,000 outlets in total. Tchibo has particularly high logistics demands because they offer their customers a weekly changing range of products with significant differences in value, weight, and physical dimensions. The case study describes the respective processes and identifies the most important participants.

The transition from previously manually controlled processes to autonomous control has been implemented and validated with multiagent-based simulation. Multiagent-based simulation has a high modelling accuracy because of its one-toone mapping between the real-world objects and their simulation counterparts. 
Moreover, the behaviour of autonomous logistics entities in simulation directly corresponds to their behaviour in real-world operation. The simulation is based on real process and dispatch data provided by Tchibo. The behaviour of over 11,500 shipping containers that arrive over the time-span of one year is examined. The results show that automated process control for standard cases with autonomous logistics is applicable to satisfy even the challenging logistics demands of Tchibo. Autonomous logistics entities are capable of allocating logistics resources efficiently and reliably, thereby considering all parameters defined by Tchibo. The validation reveals that autonomous logistics even exceeds the efficiency of the manual approach. In the examined application, the use of the implemented autonomous control showed savings of 2.6 million pallet-days in the warehouses of Tchibo per year by better utilising free times at the container terminals. Moreover, the automation allows human dispatchers to concentrate only on exceptional cases that are not covered by the multiagent system and, thus, provide better dispatching results in these cases than before when they had to handle them under extreme time pressure.

\section{Bibliography}

Bretzke, W.-R. (2008). Logistische Netzwerke. Heidelberg, Germany: Springer-Verlag.

Hülsmann, M. \& Windt, K. (Eds.). (2007). Understanding Autonomous Cooperation and Control in Logistics: The Impact of Autonomy on Management, Information, Communication and Material Flow. Heidelberg, Germany: Springer-Verlag.

Schuldt, A. (2010). Multiagent Coordination Enabling Autonomous Logistics. Doctoral Dissertation, Universität Bremen.

Wooldridge, M. (1999). Intelligent Agents. In G. Weiss (Ed.), Multiagent Systems. A Modern Approach to Distributed Artificial Intelligence (pp. 27-77). Cambridge, MA, USA: MIT Press.

Wooldridge, M. \& Jennings, N. R. (1999). The Cooperative Problem Solving Process. Journal of Logic \& Computation, 9 (4), 563-592. 
